\title{
ATUAÇÃO DO ENFERMEIRO DA ATENÇÃO PRIMÁRIA À SAÚDE NA ASSISTÊNCIA ONCOLÓGICA: REVISÃO INTEGRATIVA
}

\author{
Geize Rocha Macedo de Souza ${ }^{1}$, Luiza Helena de Oliveira Cazola ${ }^{2}$, Renata Palópoli Pícoli ${ }^{3}$
}

RESUMO: Objetivo: identificar a atuação do enfermeiro da Atenção Primária à Saúde na atenção oncológica, a partir de evidências na literatura científica. Método: trata-se de uma revisão integrativa, cuja coleta de dados foi realizada em outubro de 2017, por meio de quatro bases de dados, sendo identificados 117 artigos. A partir dos critérios de inclusão estabelecidos, foram selecionados sete. Resultados: os artigos permitiram organizar os resultados em três categorias temáticas: atividades assistenciais, seis (86\%); atividades de educação em saúde, quatro (57\%); e competências dos profissionais da Atenção Primária à Saúde, um (14\%). Considerações finais: para a assistência de enfermagem oncológica é substancial aliar o conhecimento com a prática clínica, centrada no holismo e no acompanhamento individualizado, de maneira a permitir o seu envolvimento em atividades assistenciais, de educação em saúde e de ações de prevenção e controle na Atenção Primária à Saúde.

DESCRITORES: Enfermagem Oncológica; Cuidados de Enfermagem; Atenção Primária à Saúde; Oncologia; Estratégia Saúde da Família.

\section{THE WORK OF PRIMARY HEALTH CARE NURSES IN ONCOLOGY CARE: AN INTEGRATIVE REVIEW}

ABSTRACT: Objective: to identify the role of the primary health care nurses in oncology care, based on scientific literature evidence. Method: this is an integrative review with data collected in October 2017, from four databases, resulting in 117 articles. Based on the established inclusion criteria, seven articles were selected. Results: the articles allowed the organization of results into three thematic categories: six health care activities $(86 \%)$; four health education activities $(57 \%)$; and one professional primary health care competence (14\%). Final considerations: in oncology nursing care, it is crucial to combine knowledge with clinical practice, centered in holism and individualized follow-up, to allow its involvement in activities related to healthcare, health education, and prevention and control actions in primary health care.

DESCRIPTORS: Oncology Nursing; Nursing Care; Primary Health Care; Oncology; Family Health Strategy.

\section{ACTUACIÓN DEL ENFERMERO DE ATENCIÓN PRIMARIA DE SALUD EN LA ATENCIÓN ONCOLÓGICA: REVISIÓN INTEGRATIVA}

RESUMEN: Objetivo: Identificar la actuación del enfermero de Atención Primaria de Salud en la atención oncológica a partir de evidencias en la literatura científica. Método: Revisión integrativa, con datos recolectados en octubre de 2017 a partir de cuatro bases de datos, identificándose 117 artículos. Considerando los criterios de inclusión establecidos, fueron seleccionados siete. Resultados: Los artículos permitieron organizar los resultados en tres categorías temáticas: actividades de atención, seis (86\%); actividades de educación en salud, cuatro (57\%); y competencias de profesionales de Atención Primaria de Salud, uno (14\%). Consideraciones finales: Para la atención de enfermería oncológica es fundamental cohesionar el conocimiento con la práctica clínica centrada en el holismo y en el seguimiento individualizado, de modo tal de permitir su involucración en actividades de atención, de educación en salud y de acciones de prevención y control en Atención Primaria de Salud.

DESCRIPTORES: Enfermería Oncológica; Atención de Enfermería; Atención Primaria de Salud; Oncología; Estrategia de Salud Familiar.

${ }^{1}$ Enfermeira. Mestre em Saúde da Família. Gerente Técnica da Vigilância do Câncer da Secretaria Municipal de Saúde Pública. Campo Grande, MS, Brasil.

${ }^{2}$ Enfermeira. Doutora em Saúde e Desenvolvimento. Docente da Universidade Federal de Mato Grosso do Sul. Campo Grande, MS, Brasil.

${ }^{3}$ Fonoaudióloga. Doutora em Saúde Pública. Pesquisadora da Fundação Oswaldo Cruz de Mato Grosso do Sul. Campo Grande, MS, Brasil.

Autor Correspondente:

Geize Rocha Macedo de Souza

Secretaria Municipal de Saúde Pública

R. José Amadei, 19 - 79091-130 - Campo Grande, MS, Brasil

E-mail: geize01@yahoo.com.br
Recebido: $27 / 02 / 2018$

Finalizado: $23 / 10 / 2018$ 


\section{- INTRODUÇÃO}

O câncer é um processo patológico, no qual as células se proliferam de maneira anormal, ignorando os sinais de regulação do crescimento no ambiente adjacente às células ${ }^{(1)}$. Essa doença ganhou relevância mundial, dadas as evidências epidemiológicas no Brasil e no mundo, sendo assim considerada um expressivo problema de saúde pública ${ }^{(2)}$. Classificada como uma doença crônicodegenerativa, tem como destaque a urbanização, a industrialização e o aumento da expectativa de vida como os principais fatores que contribuem para o aumento de sua incidência no mundo ${ }^{(3)}$.

A Organização Mundial da Saúde (OMS) estima para o mundo que, em 2030, serão 27 milhões de casos incidentes de câncer, 17 milhões de mortes pela doença e 75 milhões de pessoas vivas, por ano, com câncer ${ }^{(4)}$.

Neste cenário, os cuidados aos pacientes com câncer devem contemplar todos os níveis de assistência, desde a atenção básica até a especializada, para que ocorram ações de promoção, prevenção, diagnóstico, tratamento, reabilitação e cuidados paliativos ${ }^{(5)}$.

Dos três níveis de atenção, a Atenção Primária à Saúde (APS) é reconhecida pela sua capacidade em orientar a organização do sistema de saúde, com o objetivo de criar condições favoráveis para mudança do modelo assistencial centrado na doença e hospitalização, para um modelo com foco nos determinantes de saúde, enfatizando a organização dos serviços centrados no cuidado à família, desenvolvimento do vínculo, longitudinalidade, coordenação e integralidade da atenção ${ }^{(6)}$.

Ao adotar essa concepção, o enfermeiro que atua na APS desenvolve o monitoramento das condições de saúde da população, como essência da atenção de enfermagem, seja individual ou no coletivo, intervindo nos agravos de ordem patológica ${ }^{(7)}$.

Diante disso, salienta-se a importância das ações na assistência oncológica, na qual os profissionais devem atuar no processo de saúde-doença, desde a promoção até o cuidado paliativo ${ }^{(8)}$. Portanto, cabe ao enfermeiro desempenhar assistência sistematizada, integral e contínua baseada no bem-estar para o paciente e sua família, inclusive identificando situações de vulnerabilidade durante as diferentes fases da doença neoplásica, fornecendo informações acerca do tratamento e dos possíveis efeitos colaterais, além de suprir as necessidades sócio psicológicas do paciente se alicerçando em trato humanizado(9-10).

Considerando a complexidade do conhecimento na área de oncologia, e a necessidade de qualificação dos enfermeiros para o atendimento dos usuários do Sistema Único de Saúde (SUS) com diagnóstico de câncer, ${ }^{(1-2)}$ o estudo tornou-se relevante para reflexão sobre a prática do cuidado voltada para a promoção da saúde e prevenção do câncer, além de subsidiar assistência de qualidade para o paciente com câncer e sua família. Nesse sentido, o presente artigo teve por objetivo identificar a atuação do enfermeiro da APS na atenção oncológica, a partir de evidências na literatura científica.

\section{- MÉTODO}

Trata-se de uma revisão integrativa da literatura, que consiste em sumarizar as pesquisas já concluídas sobre um determinado tema de forma sistemática.

A pesquisa seguiu os padrões de rigor metodológico propostos para a revisão integrativa da literatura, que contemplou as etapas: 1) elaboração da questão temática; 2) estabelecimento de critérios de inclusão e exclusão de estudos e busca na base de dados; 3) elaboração de um instrumento de coleta de dados para as informações-chaves a serem extraídas dos estudos; 4) avaliação dos estudos incluídos na revisão; 5) interpretação dos resultados; e 6) apresentação dos resultados evidenciados/síntese ${ }^{(11)}$.

Na primeira etapa, formulou-se a questão norteadora: qual a atuação do enfermeiro da APS na atenção oncológica segundo a literatura científica da área da saúde?

A segunda etapa envolveu a busca bibliográfica por meio das bases de dados indexadas: Medical Literature and Retrieval System Online (MEDLINE), Literatura Latino-Americana e do Caribe em Ciências 
da Saúde (LILACS), Base de Dados de Enfermagem (BDENF), Coleciona SUS, Scientific Eletronic Library Online (SciELO), através de seus formulários avançados, no mês de outubro de 2017, por meio de link disponibilizado pela Biblioteca da Universidade Federal de Mato Grosso do Sul.

Para o levantamento dos artigos foram utilizados os Descritores em Ciências da Saúde (DECS): enfermagem oncológica, cuidados de enfermagem, estratégia saúde da família, atenção primária à saúde, e o descritor não-controlado atenção oncológica. Foram realizados os cruzamentos dos descritores através do conector booleano " $A N D$ ". Estes termos, em seguida, foram combinados entre si, conforme o Quadro 1.

Quadro 1 - Combinação dos descritores. Campo Grande, MS, Brasil, 2017

\begin{tabular}{|c|c|c|}
\hline $\mathbf{1}^{\mathbf{0}}$ Descritor & $\mathbf{2}^{\mathbf{o}}$ Descritor & $\mathbf{3}^{\mathbf{0}}$ Descritor \\
\hline Enfermagem oncológica & Cuidados de enfermagem & Atenção primária à saúde \\
\hline Enfermagem oncológica & Cuidados de enfermagem & Estratégia saúde da família \\
\hline Enfermagem oncológica & Atenção primária à saúde & - \\
\hline Enfermagem oncológica & Estratégia saúde da família & - \\
\hline Atenção oncológica & Atenção primária à saúde & - \\
\hline Atenção oncológica & Estratégia saúde da família & - \\
\hline
\end{tabular}

Neste estudo não se utilizou palavras-chaves referentes aos sistemas corporais e da assistência hospitalar, o que ampliaria a amostra. Com o propósito de analisar a produção científica em enfermagem oncológica, na Atenção Primária à Saúde (APS), selecionou-se as palavras com maior probabilidade de contribuir para atender à questão norteadora.

Os critérios de inclusão adotados para a seleção da amostra foram: artigos disponíveis online na íntegra, nos idiomas português, inglês e espanhol, entre o período de janeiro de 2008 a agosto de 2017, nas bases de dados indexadas selecionadas, que versavam sobre a temática do câncer na atenção primária à saúde. Excluíram-se produções científicas não relacionadas com o escopo do estudo, artigos publicados em fontes que não fossem eletrônicas, disponíveis apenas em resumo, monografias, dissertações, teses, estudos de revisões integrativas, narrativas e sistemáticas, editoriais, carta ao leitor e comentários.

Identificou-se 117 artigos e, após a remoção dos 80 duplicados, 37 foram pré-selecionados pela leitura flutuante de seus títulos e resumos, destes 22 foram excluídos por não contemplar os critérios de inclusão. Dos 15 resultantes realizou-se a leitura minuciosa dos textos, e após a amostra final foi composta por sete artigos, conforme apresentado na Figura 1. 


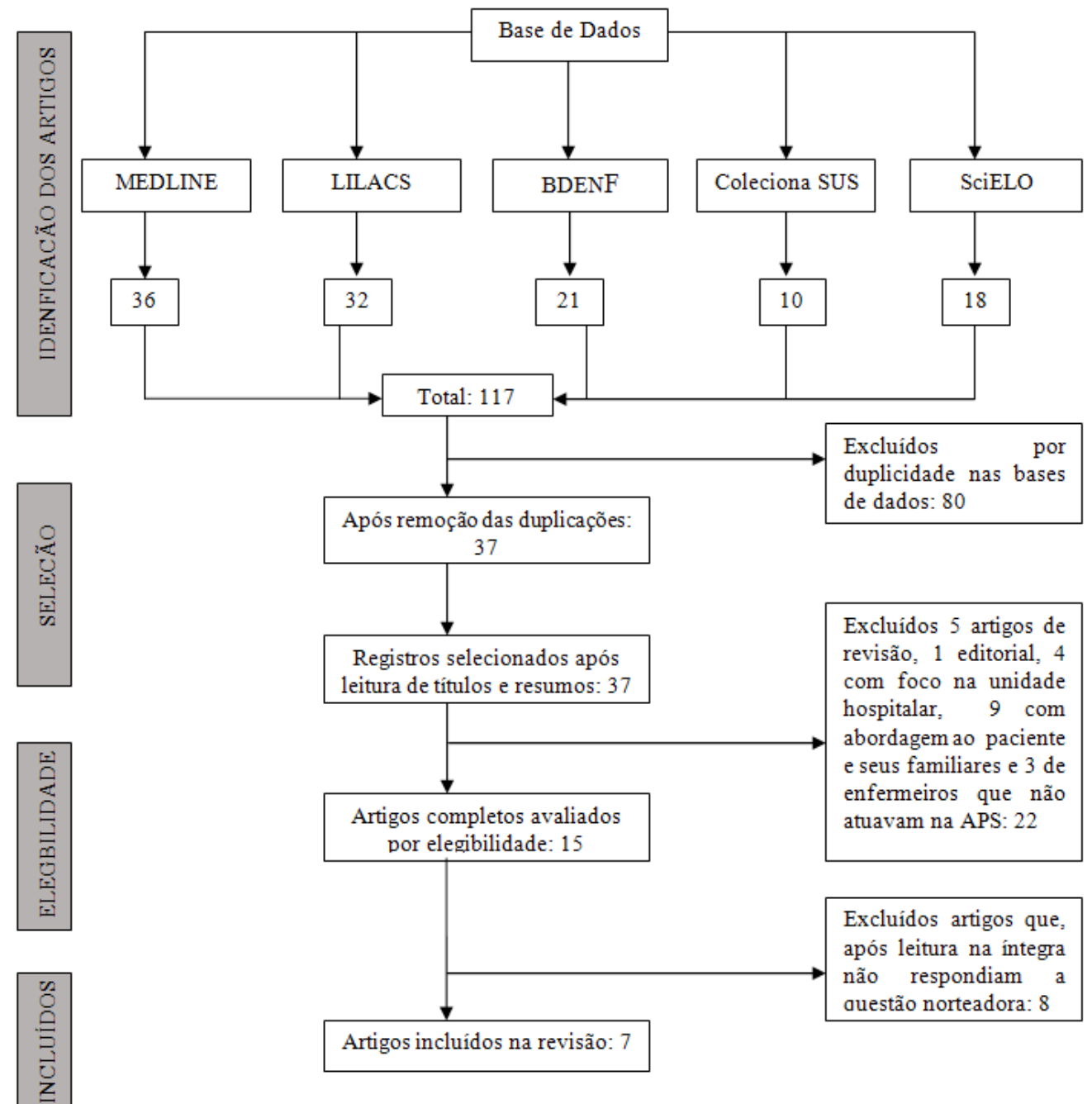

Figura 1 - Fluxograma da coleta de dados e seleção de estudos que compõe a amostra. Campo Grande, MS, Brasil, 2017

Na terceira etapa, os artigos selecionados foram organizados por meio de um instrumento de coleta de dados, de modo a facilitar o acesso, para posterior leitura integral e identificação das informações que contemplaram autoria, ano, título, objetivo, base de dados, periódico e tipo de estudo, conforme o Quadro 2.

Quadro 2 - Descrição dos artigos incluídos na revisão. Campo Grande, MS, Brasil, 2017 (continua)

\begin{tabular}{|c|c|c|c|c|c|c|}
\hline $\mathbf{N}^{\circ}$ & $\begin{array}{c}\text { Base de } \\
\text { dados }\end{array}$ & Título & Autores & $\begin{array}{c}\text { Periódico e } \\
\text { ano }\end{array}$ & Objetivo & $\begin{array}{l}\text { Tipo de } \\
\text { estudo }\end{array}$ \\
\hline 1 & SciELO & $\begin{array}{l}\text { Atuação dos } \\
\text { enfermeiros da } \\
\text { estratégia saúde da } \\
\text { família na atenção } \\
\text { oncológica }\end{array}$ & $\begin{array}{l}\text { Geize Rocha Macedo } \\
\text { de Souza; Luiza Helena } \\
\text { de Oliveira Cazola; } \\
\text { Sandra Maria do Valle } \\
\text { Leone de Oliveira }^{(12)}\end{array}$ & $\begin{array}{c}\text { Escola Anna } \\
\text { Nery } \\
2017\end{array}$ & $\begin{array}{c}\text { Conhecer a atuação } \\
\text { dos enfermeiros } \\
\text { da ESF na atenção } \\
\text { oncológica. }\end{array}$ & $\begin{array}{l}\text { Transversal } \\
\text { Quantitativo }\end{array}$ \\
\hline 2 & BDENF & $\begin{array}{l}\text { Acesso a serviços } \\
\text { de saúde para o } \\
\text { controle do câncer } \\
\text { de colo útero na } \\
\text { atenção primária }\end{array}$ & $\begin{array}{c}\text { Magna Maria Pereira } \\
\text { da Silva; Maria Tereza } \\
\text { Cicero Lagana; Clélia } \\
\text { albino Simpson; Ana } \\
\text { Michele de Farias } \\
\text { Cabral(13) }\end{array}$ & $\begin{array}{l}\text { Journal of } \\
\text { Research } \\
\text { Fundamental } \\
\text { Care on Line } \\
2013\end{array}$ & $\begin{array}{c}\text { Analisar o controle } \\
\text { do câncer do colo } \\
\text { uterino na atenção } \\
\text { básica. }\end{array}$ & Transversal \\
\hline
\end{tabular}




\begin{tabular}{|c|c|c|c|c|c|c|}
\hline 3 & LILACS & $\begin{array}{l}\text { Cuidados com } \\
\text { o paciente } \\
\text { oncológico } \\
\text { em tratamento } \\
\text { quimioterápico: o } \\
\text { conhecimento dos } \\
\text { enfermeiros da } \\
\text { estratégia saúde da } \\
\text { família }\end{array}$ & $\begin{array}{l}\text { Fernanda Strapazzon } \\
\text { da Cruz; Luciana } \\
\text { Grazziotin Rossato }^{(14)}\end{array}$ & $\begin{array}{c}\text { Revista } \\
\text { Brasileira de } \\
\text { Cancerologia } \\
2015\end{array}$ & $\begin{array}{c}\text { Identificar o nível } \\
\text { de conhecimento de } \\
\text { enfermeiros atuantes } \\
\text { na ESF. }\end{array}$ & $\begin{array}{c}\text { Transversal, } \\
\text { Prospectivo } \\
\text { e } \\
\text { quantitativo }\end{array}$ \\
\hline 4 & SciELO & $\begin{array}{c}\text { Atuação do } \\
\text { enfermeiro da } \\
\text { atenção primária } \\
\text { no controle câncer } \\
\text { e mama }\end{array}$ & $\begin{array}{l}\text { Michele de Souza } \\
\text { Teixeira; Rosely } \\
\text { Erlach Goldman; } \\
\text { Valterli Conceição } \\
\text { Sanches Gonçalves; } \\
\text { Maria GabyRivero de } \\
\text { Gutiérrez; Elisabeth } \\
\text { Níglio de Figueiredo }\end{array}$ & $\begin{array}{l}\text { Acta Paulista } \\
\text { Enfermagem } \\
\quad 2017\end{array}$ & $\begin{array}{l}\text { Analisar as ações } \\
\text { realizadas por } \\
\text { enfermeiros da } \\
\text { atenção primária } \\
\text { em saúde para } \\
\text { o rastreamento } \\
\text { oportunístico do } \\
\text { câncer de mama. }\end{array}$ & Transversal \\
\hline 5 & $\begin{array}{c}\text { Coleciona } \\
\text { SUS }\end{array}$ & $\begin{array}{l}\text { Conhecimento } \\
\text { de enfermeiros } \\
\text { da estratégia } \\
\text { saúde da família } \\
\text { sobre avaliação } \\
\text { e tratamento } \\
\text { de feridas } \\
\text { oncológicas }\end{array}$ & $\begin{array}{l}\text { Isabelle Campos de } \\
\text { Azevedo; Roberta } \\
\text { Kaliny de Souza Costa; } \\
\text { Cristyanne Samara } \\
\text { Miranda de Holanda; } \\
\text { Marina de Góes } \\
\text { Salvetti; Gilson de } \\
\text { Vasconcelos Torres }\end{array}$ & $\begin{array}{c}\text { Revista } \\
\text { Brasileira de } \\
\text { Cancerologia } \\
2014\end{array}$ & $\begin{array}{c}\text { Identificar as } \\
\text { dificuldades } \\
\text { enfrentadas pelos } \\
\text { enfermeiros no } \\
\text { cuidado às pessoas } \\
\text { com feridas } \\
\text { oncológicas no } \\
\text { contexto da ESF. }\end{array}$ & $\begin{array}{c}\text { Descritivo } \\
\text { Quantitativo }\end{array}$ \\
\hline 6 & $\begin{array}{c}\text { Coleciona } \\
\text { SUS }\end{array}$ & $\begin{array}{l}\text { O enfermeiro na } \\
\text { prevenção do } \\
\text { câncer do colo do } \\
\text { útero: o cotidiano } \\
\text { da atenção } \\
\text { primária }\end{array}$ & $\begin{array}{c}\text { Maria Carmen Simões } \\
\text { de Melo; Franciane } \\
\text { Vilela; Anna Maria } \\
\text { de Oliveira Salimena; } \\
\text { Ivis Emília de Oliveira } \\
\text { Souza(17) }\end{array}$ & $\begin{array}{c}\text { Revista } \\
\text { Brasileira de } \\
\text { Cancerologia } \\
2012\end{array}$ & $\begin{array}{c}\text { Analisar o } \\
\text { desenvolvimento da } \\
\text { prevenção e detecção } \\
\text { precoce do câncer } \\
\text { do colo do útero no } \\
\text { cotidiano assistencial } \\
\text { da enfermeira que } \\
\text { atua nas equipes de } \\
\text { ESF. }\end{array}$ & $\begin{array}{c}\text { Exploratório } \\
\text { Descritivo } \\
\text { Qualitativo }\end{array}$ \\
\hline 7 & BDENF & $\begin{array}{l}\text { A política nacional } \\
\text { de atenção } \\
\text { oncológica } \\
\text { e o papel da } \\
\text { atenção básica } \\
\text { na prevenção e } \\
\text { controle do câncer }\end{array}$ & $\begin{array}{c}\text { Roberto Parada; } \\
\text { Mônica de Assis; } \\
\text { Ronaldo Corrêa } \\
\text { Ferreira da Silva; Maria } \\
\text { Fátima Abreu; Marcos } \\
\text { André Felix da Silva; } \\
\text { Maria Beatriz Kneipp } \\
\text { Dias; Jeane Glaucia } \\
\text { Tomazelli(18) }\end{array}$ & $\begin{array}{c}\text { Revista APS } \\
2008\end{array}$ & $\begin{array}{c}\text { Oferecer uma } \\
\text { visão panorâmica } \\
\text { da estruturação } \\
\text { do controle de } \\
\text { câncer no Brasil e } \\
\text { discutir o papel da } \\
\text { atenção primária } \\
\text { na prevenção dos } \\
\text { cânceres de mama e } \\
\text { do colo do útero. }\end{array}$ & Atualização \\
\hline
\end{tabular}

$\mathrm{Na}$ quarta etapa, os artigos foram agrupados conforme suas similaridades e na seguinte, envolveu uma análise criteriosa dos estudos, e formaram-se as categorias temáticas da pesquisa, prosseguindose com discussão fundamentada a partir da literatura pertinente. Para finalizar, na sexta etapa foi processada a síntese, pela estatística descritiva.

\section{RESULTADOS}

A amostra da revisão foi composta por sete estudos que descrevem atividades ou propõem intervenções a serem realizadas pelos enfermeiros da atenção primária à saúde, na assistência ao paciente oncológico, conforme apresentado no Quadro 1.

Na identificação das fontes para a localização dos artigos, os de números 1 e 4 encontraram-se na base de dados SciELO, os artigos 2 e 7 são provenientes da BDENF, os 5 e 6 foram encontrados na base de dados Coleciona SUS e o de número 3 na LILACS. As palavras-chaves mais utilizadas pelos 
autores foram "enfermagem oncológica", presente em cinco artigos, e "atenção primária à saúde" em três artigos.

Foram encontrados sete (100\%) periódicos: três $(42,8 \%)$ da Revista Brasileira de Cancerologia; um (14,3\%) da Escola Anna Nery; um (14,3\%) da Revista de APS; um (14,3\%) do Journal of Research Fundamental Care on Line e um (14,3\%) da Acta Paulista de Enfermagem.

Em relação ao ano, verificou-se que os intervalos entre as publicações ocorreram de 2008 a 2017, com predomínio a partir de 2012, o que demonstrou o interesse pela temática gradativo e recente.

$\mathrm{Na}$ análise do delineamento dos estudos, identificaram-se quatro com métodos transversais e dois descritivos, sendo um de abordagem exploratória e qualitativo e outro quantitativo.

Os achados foram agrupados de acordo com as similaridades das atividades desenvolvidas. Desta análise, foram formuladas as categorias temáticas para realizar a síntese do conhecimento da revisão integrativa, apresentada a seguir: 1) atividades assistenciais, 2) atividades de educação em saúde e 3) competências dos profissionais da APS.

\section{Categoria 1 - Atividades assistenciais}

Nesta categoria foram incluídas atividades descritas em seis (86\%) artigos $(1,2,3,4,5,6)$ que compuseram a amostra. As atividades assistenciais realizadas pelos enfermeiros da atenção primária à saúde na assistência oncológica foram diversificadas conforme o tipo de rastreamento ou de câncer que o paciente possuía. As atividades citadas foram a realização de procedimentos em cinco (83\%) estudos $(2,3,4,5,6)$, consulta de enfermagem em três $(50 \%)(1,4,6)$, atividades de rastreamento em três $(50 \%)(2,4,6)$, acompanhamento em três $(50 \%)(1,4,6)$, encaminhamentos para equipe multidisciplinar, para complementação diagnóstica e atenção secundária em três $(50 \%)(3,4,6)$, visitas domiciliares, atendimento aos familiares e cuidados paliativos em um (17\%) (1).

\section{Categoria 2 - Atividades de educação em saúde}

Essa atividade foi encontrada em quatro (57\%) artigos $(1,4,5,6)$, sendo realizada de forma coletiva ou individual para pacientes e seus familiares/cuidadores dois $(50 \%)(1,4)$. Os temas das atividades educativas percorreram a assistência oncológica de forma variada, voltados para cuidados com o paciente e sobre a saúde do cuidador um (25\%) (1), para a prevenção do câncer de mama um (25\%) (4), para a higienização do paciente e cuidados com a ferida oncológica um (25\%) (5), para quebra de tabus a respeito do exame colpocitológico, a fim de sensibilizar a mulher para sua realização com regularidade um (25\%) (6).

\section{Categoria 3 - Competências dos profissionais da APS}

Nessa categoria foi encontrado um (14\%) artigo (7), que descreve todas as atividades que devem ser realizadas pelos profissionais para a prevenção e controle do câncer na APS.

\section{- DISCUSSÃO}

A enfermagem tem em sua essência o compromisso com a integralidade, o qual se concretiza pelo acolhimento, vínculo e diálogo ${ }^{(19)}$ e se sustenta na identificação das necessidades de cuidados referidas pelos pacientes e familiares, por ser uma prática centrada na pessoa e não apenas no procedimento ${ }^{(20)}$.

A assistência aos pacientes com câncer requer habilidades técnico-científicas dos enfermeiros de modo a transmitir maior eficiência no cuidado ao tratamento, devendo conhecer a doença, seus prognósticos, cuidados de suporte e terminais, pois estes se encontram inclusive no domicílio ${ }^{(3,21)}$.

É sabido que a assistência oncológica prestada pelos enfermeiros da APS é multifária. O câncer é uma 
doença com acompanhamento previsto pela APS, apontado pela Política Nacional para a Prevenção e Controle do Câncer, na Rede de Atenção à Saúde das Pessoas com Doenças Crônicas, no âmbito do SUS ${ }^{(5)}$.

São imperiosas as atividades exercidas pelo enfermeiro na APS na prevenção do câncer e assistência ao paciente oncológico, como: acolhimento, consulta de enfermagem, visita domiciliar, orientações ao paciente e família sobre os cuidados paliativos, quimioterapia e radioterapia, planejamento e execução de ações de rastreamento como citologia em mulheres de faixa etária prioritária, exame clínico das mamas, acompanhamento de pacientes tratados ou em tratamento, avaliação e indicação de terapêutica para feridas oncológicas, curativos, solicitação de exames, encaminhamentos, apoio terapêutico, e promoção da saúde no âmbito individual e coletivo, ${ }^{(13-18)}$ atividades estas semelhantes com um estudo realizado com enfermeiros de atenção básica de Florianópolis ${ }^{(2)}$.

Das inúmeras atribuições do enfermeiro na APS, vale ressaltar a consulta de enfermagem, uma atividade exclusiva deste profissional, ${ }^{(22)}$ capaz de elucidar dúvidas comuns e isoladas de pacientes que iniciam o processo terapêutico, constituindo-se como momento oportuno para o melhor entendimento do paciente sobre a doença ${ }^{(23)}$. Além de contribuir para a redução dos índices de depressão, fadiga, distúrbios do padrão do sono, stress, nível da dor, assegurando a qualidade de vida e o bem-estar dos pacientes, ${ }^{(24)}$ porém no estudo realizado em um município do sul do Brasil, a consulta de enfermagem ao paciente oncológico não foi citada como demanda de atendimento dos enfermeiros da atenção básica $^{(2)}$.

O enfermeiro da APS, agente de promoção da saúde, deve sistematizar a assistência para o indivíduo, a família ou a comunidade no ambiente em que vive ${ }^{(25)}$. A promoção da saúde abrange o fortalecimento da capacidade individual e coletiva das pessoas trabalharem com a diversidade das causas saúde-doença, estimulando a autonomia do cidadão e da comunidade ${ }^{(26)}$.

Os profissionais de saúde constituem peça fundamental para o enfrentamento do câncer, visto que, diante do diagnóstico de uma doença, os pacientes e familiares expressam interesse em ter conhecimento acerca da doença e dos potenciais efeitos colaterais que o tratamento pode trazer ${ }^{(23,27)}$. O cuidado do enfermeiro à pessoa com câncer não pode limitar-se à terapêutica do paciente, é preciso que se amplie a visão para os familiares, ofertando condições de bem-estar, prover conforto, cuidados básicos e fisiopatológicos, além de dar atenção aos anseios, desejos e vontades dos pacientes e familiares $^{(28)}$.

Os estudos quatro e cinco apresentaram adesão pelos enfermeiros na realização de práticas educativas, e o estudo seis salientou a necessidade de potencializar as atividades educativas e que estas devem ser elaboradas e praticadas por todos os membros da APS $(4,5,6)$. São indispensáveis investimentos em ações educativas que tragam a prática humanizada e que resultem em impacto sobre a compreensão quanto à necessidade de prevenção ${ }^{(29)}$. A equipe de enfermagem atua diretamente com ações educativas principalmente na APS, pois é habilitada e capacitada para cuidar do usuário e da comunidade, levando em consideração as necessidades curativas, preventivas, bem como o controle das doenças crônicas através de ações de educação em saúde ${ }^{(30)}$.

O trabalho do enfermeiro é difícil de ser desenvolvido, considerando que há necessidade de competências e habilidades específicas para gerenciar a complexidade que envolve o processo laboral da saúde e da enfermagem ${ }^{(31)}$. O estudo sete apresentou as atribuições específicas e comuns de todos os profissionais da APS, destacando a importância dos profissionais na assistência aos indivíduos sob tratamento e em estágio terminal, além das atividades de promoção da saúde e prevenção da doença ${ }^{(18)}$.

As atividades de enfermagem assistenciais para pacientes em cuidados paliativos ou estágio terminal deverão estar direcionadas para a avaliação sistemática dos sinais e sintomas, priorizando as necessidades de cada paciente, bem como na interação da dinâmica familiar, através da comunicação, agregando as orientações, a fim de alcançar os objetivos terapêuticos ${ }^{(32)}$.

Para potencializar a assistência oncológica, em 2013 o Ministério da Saúde instituiu a Política Nacional para a Prevenção e Controle do Câncer na Rede de Atenção à Saúde das Pessoas com Doenças Crônicas no âmbito do SUS, a qual propõe a formação de profissionais e a promoção de educação permanente, por meio de atividades que visem à aquisição de conhecimentos, habilidades e atitudes para qualificação do cuidado ao paciente oncológico. Para tanto, as atividades de educação 
permanente podem assegurar a manutenção da competência da equipe de enfermagem em relação ao cuidado dos usuários do SUS na atenção básica e no contexto da oncologia ${ }^{(33)}$.

O estudo possui como limitação o fato de possuir um número reduzido de artigos encontrados sobre a questão norteadora nas bases eletrônicas, sendo fundamental que os enfermeiros da APS tornem públicas as experiências na assistência ao paciente oncológico, em forma de publicações científicas e utilizem de forma adequada os descritores para que a busca seja ampliada.

\section{CONCLUSÃo}

Para a assistência de enfermagem oncológica, é substancial aliar o conhecimento com a prática clínica, centrada no holismo e no acompanhamento individualizado, capaz de garantir um atendimento efetivo. A pesquisa nacional nessa área está sendo construída progressivamente, de modo que há necessidade dos enfermeiros e pesquisadores desenvolverem estudos na área da oncologia na APS, a fim de colaborar com a construção do conhecimento e subsidiar ações que visem melhor assistência e qualidade de vida do paciente com câncer.

A APS tem ampla magnitude de atuação para a consolidação da Política Nacional para Prevenção e Controle do Câncer, buscando integrar os diferentes níveis de assistência aos usuários com câncer, além de prover ações de promoção da saúde e prevenção do câncer. O enfermeiro é integrante ativo nesse processo, atuando com responsabilidade e sensibilidade em cada etapa e assim qualificando a assistência oncológica.

A análise dos estudos selecionados demonstrou a importância do envolvimento do enfermeiro na assistência de enfermagem ao paciente oncológico, que deve atuar em atividades assistenciais, de educação em saúde e de ações de prevenção e controle do câncer na Atenção Primária à Saúde.

\section{REFERÊNCIAS}

1. Santos TRA, Souza SR. Nursing interventions for children and adolescents with cancer during the chemotherapy treatment. J. res. fundam. care. [Internet].2015 [acesso em2016 jun 15];7(3). Disponível em: http://dx.doi. org/10.9789/2175-5361.2015.v7i3.2853-2864.

2. Rosa LM, Souza AIJ, Anders JC, Silva RN, Silva GS, Fontão MC. Demandas de atendimento de enfermagem e de qualificação em oncologia na atenção básica em saúde. Cogitare enferm. [Internet]. 2017 [acesso em 2017 maio 29];22(4). Disponível em:http://dx.doi.org/10.5380/ce.v22i4.51607.

3. Belhiane HPP, de Matos LRP, Camargos F. O paciente frente ao diagnóstico de câncer e atuação dos profissionais de enfermagem: uma revisão integrativa de literatura. R. Enferm. Cent. O. Min. [Internet] 2014;4(3) [acesso em 11 jun 2017]. Disponível: http://dx.doi.org/10.19175/recom.v0i0.592.

4. Forman D, Feriay J. The global and regional burden of cancer. In: Stewart BW, Wild CP, editores. World Cancer Report 2014. Lyon: InternationalAgency for ResearchonCancer; 2014. p. 16-53.

5. Ministério da Saúde (BR). Portaria n. 874, de 16 de maio de 2013. Institui a Política Nacional para a Prevenção e Controle do Câncer na Rede de Atenção à Saúde das Pessoas com Doenças Crônicas no âmbito do Sistema Único de Saúde (SUS).Diário Oficial da União, Brasília, 17 mai. 2013. Seção 1.

6. Arantes LJ, Shimizu HE, Merchán-Hamann E. Contribuições e desafios da Estratégia Saúde da Família na Atenção Primária à Saúde no Brasil: revisão da literatura. Cienc. saude colet. [Internet]. 2016 [acesso em 2017 jul 01];21(5) Disponível em: http://dx.doi.org/10.1590/1413-81232015215.19602015.

7.Costa EMS, Peres AM, Bernardino E, Sade PMC. Estilos de liderança dos enfermeiros que atuam na Estratégia Saúde da Família. Ciênc. cuid. saúde.[Internet]. 2015 [acesso em 2017 jul 02];14(1). Disponível em: http://dx.doi. org/10.4025/cienccuidsaude.v14i1.20756.

8. Cavalcante SAM, Silva FB, Marques CAV, de Figueiredo EM, de Gutiérrez MGR. Ações do Enfermeiro no rastreamento e Diagnóstico do Câncer de Mama no Brasil. Rev. bras. cancerol. [Internet]. 2013 [acesso em 2017 
jul 03];59(3). Disponível em: http://www.inca.gov.br/rbc/n_59/v03/pdf/17-revisao_literatura-acoes-enfermeirorastreamento-diagnostico-cancer-mama-brasil.pdf.

9. da Mata LRF, Cunha AC, Ziviani CSL, Fonseca TG, Bernardes MFVG,Oliveira PP. Morbidade psicológica e implicações para a recuperação de adultos após cirurgia oncológica. CogitareEnferm. [Internet] 2018;23(1) [acesso em 29 mai 2018]. Disponível: http://dx.doi.org/10.5380/ce.v23i1.53089.

10. Delfino CTA, Ferreira WFS, Oliveira EC, Dutra DA. Câncer infantil: atribuições da enfermagem em cuidado paliativo. Revista Saúde e Desenvolvimento [Internet]. 2018 [acesso em 2018 maio 29];12(10). Disponível em:https:// www.uninter.com/revistasaude/index.php/saudeDesenvolvimento/article/view/866/497.

11. Mendes KDS, Silveira RCCP, Galvão CM. Revisão integrativa: método de pesquisa para a incorporação de evidências na saúde e na enfermagem. Texto contexto-enferm[Internet]. 2008 [acesso em2017 jun 03];17(4). Disponível em:http://dx.doi.org/10.1590/S0104-07072008000400018.

12. Souza GRM, Cazola LHO,Oliveira SMVL. Work of family health strategy nurses in oncology care. Esc. Anna Nery [Internet].2017 [acesso em2017 ago 25];21(4). Disponível em: http://dx.doi.org/10.1590/2177-9465-ean-2016-0380.

13. Silva MMP, Lagana MTC, Simpson CA, Cabral AMF. Access to health services for the control of cancer of the uterine cervix in primary care. J. res. fundam. care. [Internet]. 2013 [acesso em2017 ago 10];5(3). Disponível em: http://dx.doi.org/10.9789/2175-5361.2013v5n3p273.

14. Cruz FS, Rossato LG. Cuidados com o Paciente Oncológico em Tratamento Quimioterápico: o Conhecimento dos Enfermeiros da Estratégia Saúde da Família. Rev. bras. cancerol. [Internet]. 2015 [acesso 2017 ago 03];61(4). Disponível em: http://www.inca.gov.br/rbc/n_61/v04/pdf/04-artigo-cuidados-com-o-paciente-oncologico-emtratamento-quimioterapico-o-conhecimento-dos-enfermeiros-da-estrategia-saude-da-familia.pdf.

15. Texeira MS, Goldman RE, Gonçalves VCS, de Gutiérrez MGR, de Figueiredo EM.Atuação do enfermeiro da Atenção Primária no controle do câncer de mama. [Internet]. 2017 [acesso em2017 ago 05];30(1). Disponível em: http://dx.doi.org/10.1590/1982-0194201700002.

16. Azevedo IC, Costa RKS, de Holanda CSM, Salvetti MG, Torres GV. Conhecimento de Enfermeiros da Estratégia Saúde da Família sobre Avaliação e Tratamento de Feridas. Rev. bras. cancerol. [Internet]. 2014 [acesso em 2017 ago10];60(2). Disponível em: http://www.inca.gov.br/rbc/n_60/v02/pdf/05-artigo-conhecimento-de-enfermeirosda-estrategia-saude-da-familia-sobre-avaliacao-e-tratamento-de-feridas-oncologicas.pdf.

17. Melo MCSC, Vilella F,Salimena AMO, Souza IEO. O Enfermeiro na Prevenção do Câncer do Colo do Útero: o Cotidiano da Atenção. Rev. bras. cancerol. [Internet]. 2012 [acesso em 2017 ago 15];58(3). Disponível em: http:// www1.inca.gov.br/rbc/n_58/v03/pdf/08_artigo_enfermeiro_prevencao_cancer_colo_utero_cotidiano_atencao_ primaria.pdf.

18. Parada R, Assis M, da Silva RCF, Abreu MF, Silva MAF, Dias MBK, et al. A política nacional de atenção oncológica e o papel da atenção básica. Rev. APS [Internet]. 2008 [acesso em 2017 ago 20];11(2). Disponível em: https://aps. ufjf.emnuvens.com.br/aps/article/view/263/100.

19. Backes DS, Pereira AD, Marchiori MT, Rupolo I, Backes MTS, Büscher A. Vínculo profissional- usuário: competência para a atuação na Estratégia Saúde da Família. Av. Enferm. [Internet]. 2015 [aceso em 2017 ago 30]; 33(2). Disponível em: http://dx.doi.org/10.15446/av.enferm.v33n2.50418.

20. Pereira DG, Fernandes J, Ferreira LS, Rabelo RO, Pessalacia JDR, Souza RS. Significados dos cuidados paliativos na ótica de enfermeiros e gestores da Atenção Primária à Saúde. Rev. enferm. UFPE on line.[Internet]. 2017 [acesso em 2017 set 01];11(Suppl 3). Disponível em: https://periodicos.ufpe.br/revistas/revistaenfermagem/ article/viewFile/13977/16825.

21. Marchi JA, Paula CC, Girardon-Perlini NMO, Sales CA. Significado de ser-cuidador de familiar com câncer e dependente: contribuições para a paliação. Texto contexto-enferm[Internet]. 2016 [acesso em 2017 set 05];25(1). Disponível em:http://dx.doi.org/10.1590/0104-07072016007600014.

22. Conselho Federal de Enfermagem. Lei n. 7498, de 25 de junho de 1986. Dispõe sobre a regulamentação do exercício da Enfermagem e dá outras providências. Rio de Janeiro: COFEN; 1986.

23. Andrade KBS, Francz ACL, Grellmann MS, Belchior PC, Oliveira JA, Wassita DN. Consulta de enfermagem: 
avaliação da adesão ao autocuidado dos pacientes submetidos à radioterapia.Rev. enferm. UERJ[Internet]. 2014 [acesso em 2017 set 10];22(5). Disponível em: http://dx.doi.org/10.12957/reuerj.2014.11227.

24. Ramos AF, Fonseca C, Coelho I, Guia S, Santos V. Indicadores sensíveis aos cuidados de enfermagem na pessoa em situação oncológica: revisão sistemática da literatura. J Aging Innovation [Internet]. 2015 [acesso em2017 set 11];5(1). Disponível em: http://www.journalofagingandinnovation.org/wp-content/uploads/2-Enfemagemindicadores-oncologia.pdf.

25. Varela GC, Fernandes SCA. Conhecimentos e práticas sobre a sistematização da assistência de enfermagem na Estratégia Saúde da Família.Cogitare enferm. [Internet]. 2013 [acesso em2017 set 05];18(1). Disponível em: http://dx.doi.org/10.5380/ce.v18i1.31317.

26. Souza MG, Mandu ENT, Elias AN. Percepções de enfermeiros sobre seu trabalho na Estratégia Saúde da Família. Texto contexto- enferm[Internet].2013 [acesso em2017 set 12];22(3). Disponível em: http://dx.doi. org/10.1590/S0104-07072013000300025.

27. Lageman SK, Brown PD, Anderson SK, Lachance DH, Yan E, Laack NNI, et al. Exploring primary brain tumor patient and caregiver needs and preferences in brief educational and support opportunities. Support Care Cancer[Internet]. 2015 [acesso em 2017 out 10]23(3). Disponível em: https://doi.org/10.1007/s00520-014-2413-y.

28. Brandão MCP, Anjos KF, Sampaio KCP, Mochizuki AB, Santos VC. Cuidados paliativos do enfermeiro ao paciente oncológico. REBRASF. [Internet] 2017 [acesso em 2018 maio 19];1(2). Disponível em:http://www.seeradventista.com.br/ojs/index.php/RBSF/article/view/879/743.

29. Oliveira FB, Custódio DM, Hollanda IS, Boas PGV, Caliman LP. Ações educativas e a prevenção do câncer de colo uterino na atenção básica. Rev. APS [Internet]. 2016 [acesso em 15 out 2017];19(2). Disponível em: https://aps. ufjf.emnuvens.com.br/aps/article/view/3238/983.

30. Azevedo PR, de Souza MM, Souza NF, Oliveira SHS. Ações de educação em saúde no contexto das doenças crônicas: revisão integrativa. J. res. fundam. care. [Internet]. 2018 [acesso em 2017 maio 30];10(1). Disponível em: http://dx.doi.org/10.9789/2175-5361.2018.v10i1.260-267.

31. Pires AS, Souza NVDO, Penna LHG, Tavares KFA, D'oliveira CAFB, Almeida CM. A formação de enfermagem na graduação: uma revisão integrativa da literatura.Rev. enferm. UERJ. [Internet]. 2014 [acesso em 2017 nov 01];22(5). Disponível em:http://dx.doi.org/10.12957/reuerj.2014.11206.

32. Luiz MM, Mourão JJN, Vasconcelos AKB, Brito MCC. Cuidados paliativos em enfermagem ao idoso em UTI: umarevisão integrativa. J. res. fundam. care. [Internet].2018 [acesso em 2017 maio 30];10(2). Disponível em: http:// dx.doi.org/10.9789/2175-5361.2018.v10i2.585-592.

33. Rosa LM, de Souza AIJ, Anders JC, da Silva RN, Silva GS, Fontão MC. Demandas de atendimento de enfermagem e de qualificação em oncologia na atenção básica em saúde. Cogitare enferm.[Intenet]. 2017 [acesso em2017 nov 10];(22)4. Disponível em: http://dx.doi.org/10.5380/ce.v22i4.51607. 